\title{
A Novel RF Excited Plasma Cathode Electron Beam Gun Design
}

\author{
Sofia del Pozo, Graduate Member, IEEE, Colin N. Ribton, and David R. Smith
}

\begin{abstract}
This paper presents a new radio frequency (RF) excited plasma-cathode electron beam (EB) gun design and experimental results at a frequency of $84 \mathrm{MHz}$. The design offers the following benefits over thermionic-cathode triode EB guns: unlimited cathode lifetime and as a result reduced maintenance costs; no requirement for a grid electrode, avoiding beam aberration; and rapid beam pulsing. The construction of the diode gun was completed and the results of this work demonstrate that the electron beam can be switched on in $200 \mathrm{~ns}$ and off within 800 ns. Electrons were extracted from a 2-3 mm plasma chamber and then accelerated by an electric field applied in a vacuum chamber at $10^{-5}$ to $10^{-6}$ mbar producing a collimated electron beam. The ionized gas used was nitrogen at a 0.5 to 1 bar pressure. The EB gun has been operated at $-60 \mathrm{kV}$ accelerating potential and has produced beams of up to $3.2 \mathrm{~kW}$ power continuous wave (CW). Modulation of the RF signal was used to control the beam power. Details of the design features of the plasma device are given and evidence of the advantages over conventional EB guns is provided through empirical results.
\end{abstract}

Index Terms-Amplitude modulation, electron sources, plasma devices, pulsed power systems, vacuum electronics.

\section{INTRODUCTION}

$\mathrm{T}$ HERMIONIC cathode electron beam (EB) guns are a widely used electron gun type for vacuum electron devices (such as klystrons, gyrotrons, betatrons and free electron lasers), x-ray generators and material processing equipment (e.g. welding and melting) [1]. The emitter is usually a metal cathode that is heated up until the electrons gain sufficient energy to leave the surface, which are then accelerated by a high gradient electric field to form a beam. However, cathode lifetime is limited due to material evaporation [2, 3] and erosion such that frequent maintenance is required. The beam current from diode electron guns can only be changed slowly (i.e. of the order $100 \mathrm{~ms}$ ) due to the thermal inertia of the cathode. Consequently, grids are often used to control beam current more rapidly, but the grid electrode in an EB triode gun introduces beam aberration [3,

Manuscript received October 30, 2013.

This work was supported in part by TWI Ltd.

David R. Smith, is with the Electronic \& Computer Engineering Department, Brunel University, Uxbridge, UB8 3PH, UK. (e-mail: david.smith@brunel.ac.uk).

Colin Ribton is with the Electron Beam Processes Section at TWI Ltd, Granta Park, Great Abington, Cambridge, CB21 6AL, UK. (e-mail: colin.ribton@twi.co.uk).

Sofia del Pozo is with Brunel University and TWI Ltd. (e-mail: sofia.delpozo@affiliate.twi.co.uk).

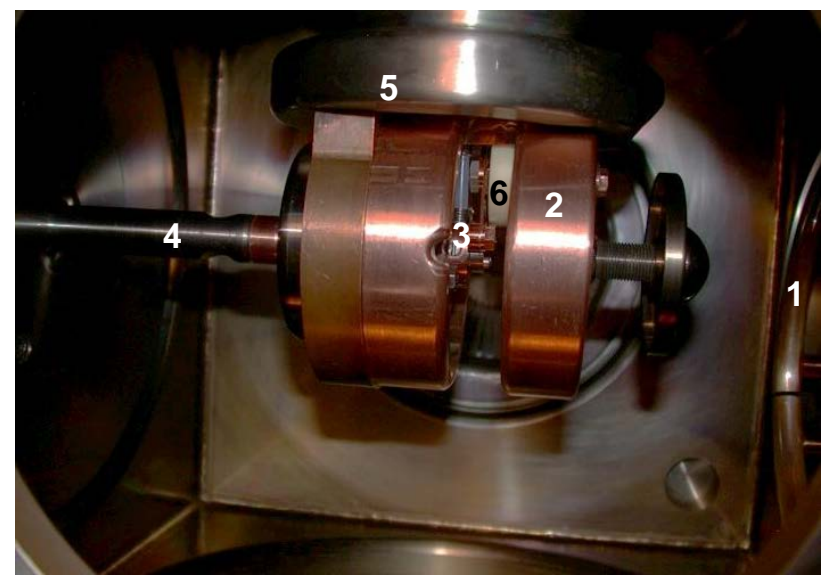

Fig. 1. Photograph showing interior of the plasma EB gun vacuum housing: a RF signal is applied to a first antenna 1, and this induces a current in the second antenna 2 , which resonates with a parallel capacitor 6 . The resonant voltage is applied across the plasma chamber. The gas fed into the chamber is controlled by a needle valve 3 . The gun is mounted on a high voltage insulator 5 at $-60 \mathrm{kV}$. The free electrons are extracted and accelerated towards the anode 4 (at $0 \mathrm{~V}$ potential).

4], which for some electron devices may affect the beam characteristics and device operation. Rapid pulsing of the beam in an EB triode gun requires complex and expensive electronic control of the grid electrode voltage and, for materials processing, pulsing at transition times below $1 \mathrm{~ms}$ is not generally available.

N. Rempe et al. [5] have presented a plasma cathode EB gun using a DC signal for the plasma excitation. The gun operates at up to $-60 \mathrm{kV}$ accelerating voltage and $12 \mathrm{~kW}$ power. However, rapid beam pulsing is not practicable due to the requirement for the cable and plasma chamber capacitances to be charged and discharged, which necessitates a high frequency response, high current drive, elevated at the accelerating voltage.

This paper presents work on a plasma-cathode [6-8] gun design resonating at a frequency of $84 \mathrm{MHz}$ and capable of producing beams of up to $3.2 \mathrm{~kW}$ power constant wave (CW). Electrons are extracted from a plasma source instead of an emissive material surface allowing electron beam parameters to be stable over a long time [6]. Plasma-cathode guns have much longer lives than guns with standard cathode assemblies [9]. The plasma-cathode provides solutions to the main problems encountered in thermionic-cathode EB guns [vide supra]. Fig. 1 is a photograph of the EB gun apparatus developed. 
Key features of the plasma electron source design are given in Section II. Section III describes the methodology of the work. Section IV gives a summary of the results obtained in switching tests at $-25 \mathrm{kV}$ accelerating voltage and $84 \mathrm{MHz}$ resonant frequency, and the plasma electron source is compared to other electron sources. Section V contains conclusions and future work.

\section{DESIGN CONCEPT}

The plasma EB gun consists of a plasma chamber used as a cathode or electron source, an RF plasma generation unit, and a particle-accelerating unit for extracting the electrons from the plasma chamber and accelerating them to form a beam.

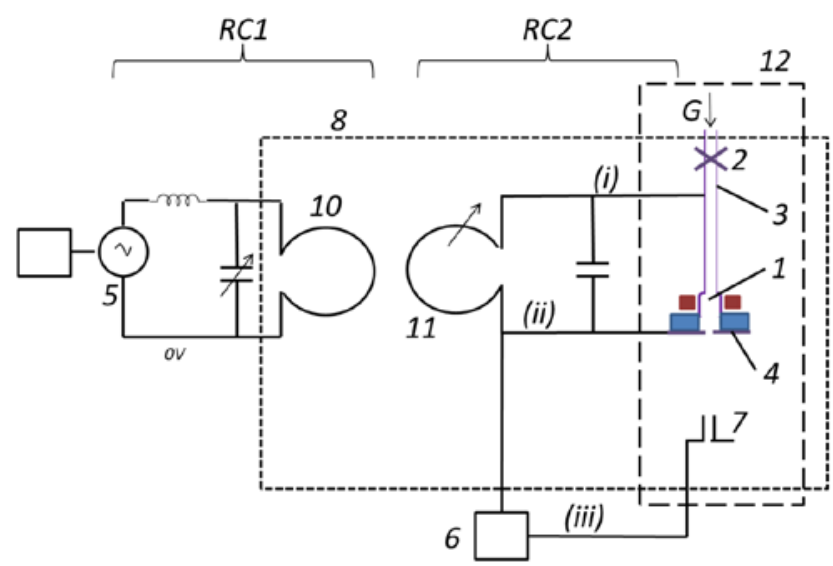

Fig. 2. Circuit schematic of the plasma gun showing electrical circuit and electrodes [10].

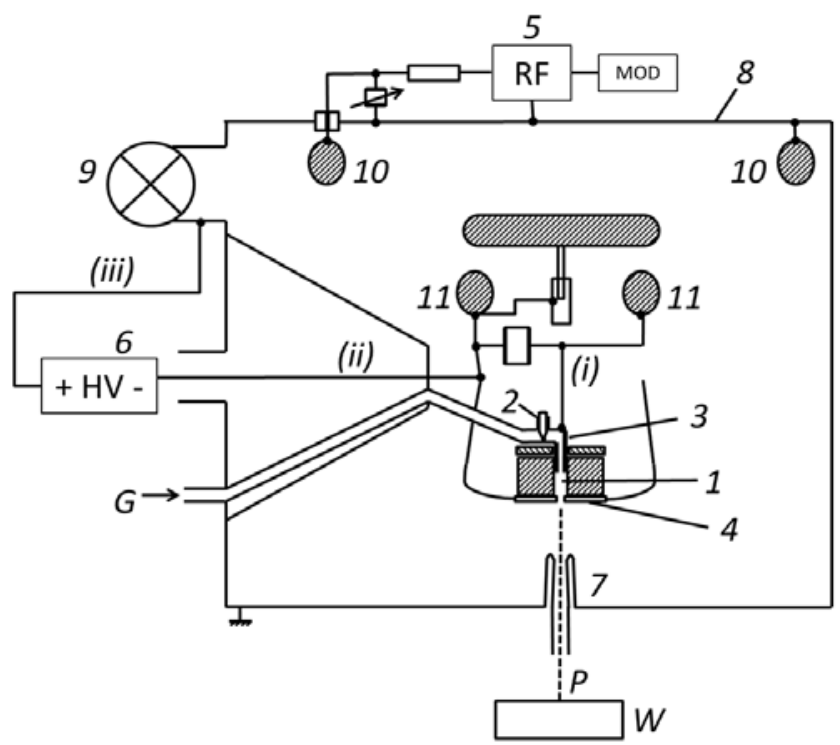

Fig. 3. Schematic cross-section of the EB gun design showing an electron beam (P) extracted from the plasma chamber (1) and accelerated towards a work piece $(\mathrm{W})[10]$.

Fig. 2 shows a circuit schematic of the plasma cathode EB gun and Fig. 3 is a schematic cross section of it, both of the figures comprising [10]: a plasma chamber (1) provided with an inlet (2) for the controlled ingress of gas $(G)$, a hollow electrode (3) and a diaphragm (4) with an aperture for the extraction of electrons from it; a radiofrequency (RF) plasma generation unit consisting of an RF power supply (5) (up to $50 \mathrm{~W}$ ) inductively coupled to the plasma chamber by a pair of resonant circuits (RC) (RC1 is the transmitter and RC2 is the receiver) tuned to resonate at the same resonant frequency; an electron accelerating unit consisting of a DC power source (6) applying a high voltage between the plasma chamber and an accelerating electrode (7) on lines (ii) and (iii) respectively.

The plasma chamber, accelerating unit and the resonant circuits are within a housing (8) at a pressure of $10^{-5} \mathrm{mbar}$, in order to maintain electrical isolation between the electrodes. However, the pressure inside the plasma chamber is higher ( 0.5 to $1 \mathrm{mbar}$ ). A needle valve controls the ingress of a steady low flow of gas into the chamber. Since the plasma chamber is connected to the gun housing directly through the diaphragm hole, the diameter of this aperture plays an important role in determining the pressure in the gun housing. Quantity flow rates through the inlet and the aperture are therefore preferably arranged such that the chamber pressure remains substantially constant [10]. The gun housing is constantly being evacuated by a turbo molecular pump (9) and a backing pump to keep it at the right vacuum level.

The resonant circuits are configured as parallel LC circuits. Both the first antenna (10) and the second antenna (11) are single turn inductors. The quality factor of the coupled resonant circuits is high enough (at least 500) so that the voltage of the induced RF signal is substantially higher than the output voltage on RC1. The values of the parallel capacitors were chosen so that the system is at its resonance frequency. This capacitance can be changed by using the tuning mushroom in the gun.

The plasma chamber contributes to the capacitance of the second resonant circuit. When a plasma is excited within the chamber, the loading of the secondary circuit will increase and this will lower the circuit's Q factor, lowering the plasma voltage but making more current available for sustaining the plasma. [10]

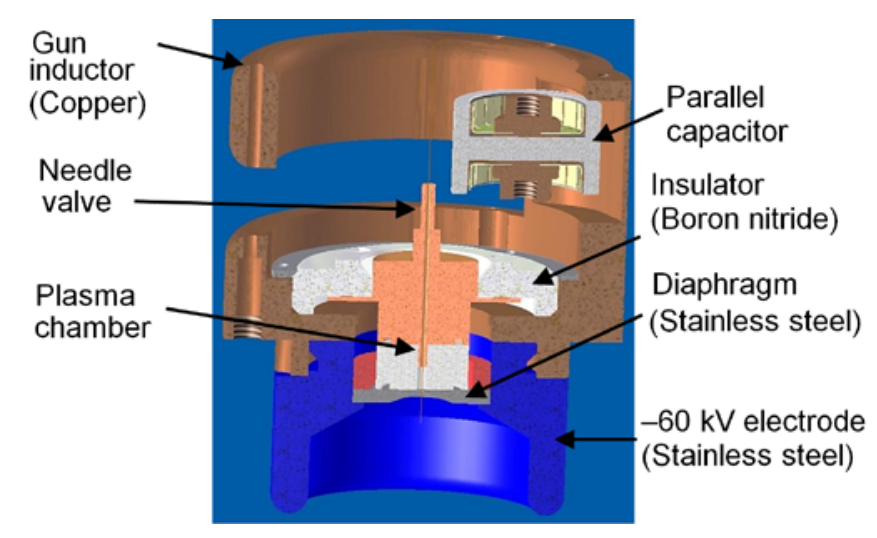

Fig. 4. Half-section of the EB gun device showing RC2, plasma chamber and $-60 \mathrm{kV}$ electrode.

Fig. 4 illustrates a half-section of the EB gun body showing RC2, the plasma chamber and the $-60 \mathrm{kV}$ electrode. Different materials were used for the diaphragm and the plasma 
chamber in order to get higher density of secondary electrons. The upper wall of the chamber and the diaphragm are formed of conductive material, and the RF voltage is applied between them. The side walls between them is formed of an insulating material (e.g. boron nitride or alumina ceramic).

\section{Methodology}

Several configurations of EB gun have been investigated. The work presented here used a configuration illustrated in Fig 5 with gun parameters given in Table 1 .

Several gases including air, neon and nitrogen, were tested for the generation of the plasma. Inert gases were favored so

TABLE I

VALUES OF THE MAIN PARAMETERS INVOLVED IN THE PLASMA-CATHODE EB GUN OPERATION

\begin{tabular}{|l|r|}
\hline RF output power amplitude & up to $50 \mathrm{~W}$ \\
\hline Resonant frequency & $84 \mathrm{MHz}$ \\
\hline Induced RF signal & $1-10 \mathrm{kV}$ \\
\hline Diaphragm aperture diameter & $0.1-1 \mathrm{~mm}$ \\
\hline Plasma chamber diameter & $<12 \mathrm{~mm}(2-3 \mathrm{~mm})$ \\
\hline Accelerating voltage & $25 \mathrm{kV}-60 \mathrm{kV}$ \\
\hline Plasma chamber pressure & $0.1 \mathrm{mbar}$ to $1 \mathrm{mbar}$ \\
\hline Gas fed pressure & $1 \mathrm{bar}$ \\
\hline
\end{tabular}

as to avoid chemical reaction with the metal parts of the plasma chamber. Noble gases were found to be readily ionized, but it was important to contain the plasma within the plasma chamber as ionization in the electron accelerator region could lead to high voltage breakdown between the EB gun and the anode [11, 12].

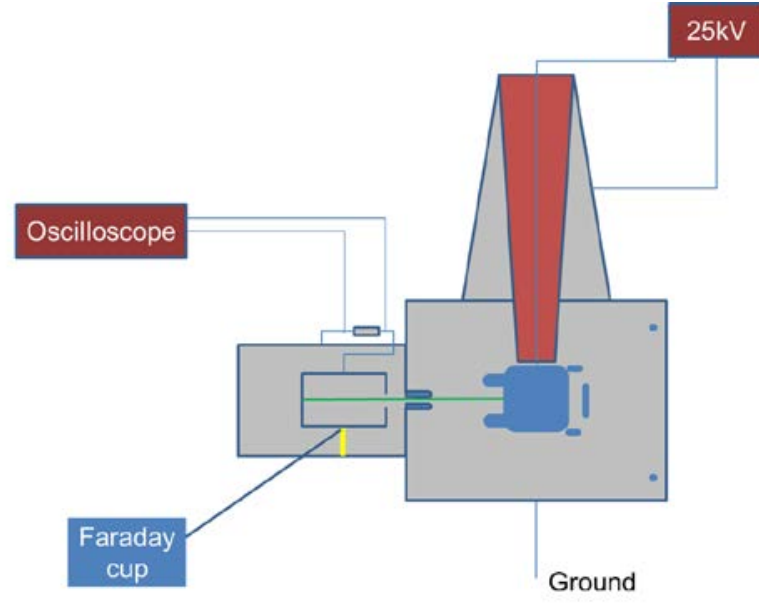

Fig. 5. $-25 \mathrm{kV}$ test set up, showing Faraday cup and resistor to measure the beam current extracted from the plasma.

The ability of the EB gun to produce a rapidly pulsed beam was tested using the configuration shown in Fig. 2. A Faraday cup was used to collect the beam current. Fig. 5 illustrates the test set up. The RF excitation of the plasma was modulated, the envelope of the RF being compared with the beam current generated by the gun.

\section{EXPERIMENTAL RESULTS}

A. Test at $-25 \mathrm{kV}$ accelerating voltage and $84 \mathrm{MHz}$ resonant frequency.

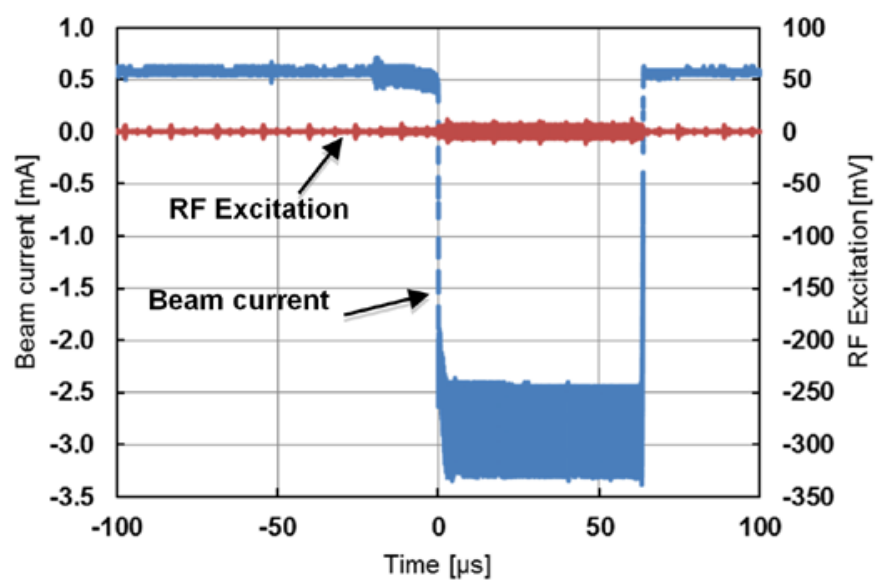

Fig. 6. Test results at $-25 \mathrm{kV}$ acceleration voltage, showing input $\mathrm{RF}$ voltage applied to RC1 and beam current from the plasma.

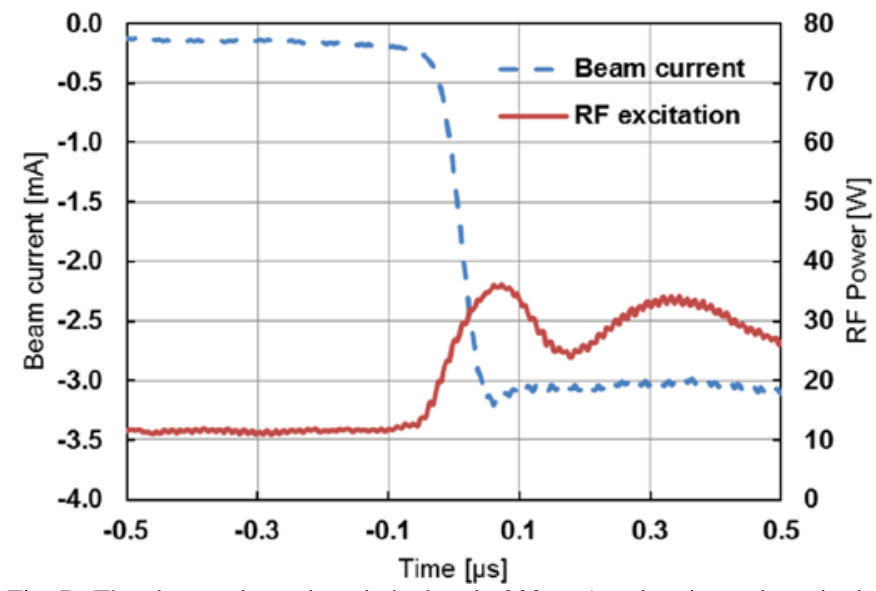

Fig. 7. The electron beam is switched on in $200 \mathrm{~ns}$ Acceleration voltage in the test is $-25 \mathrm{kV}$ and the resonant frequency is $84 \mathrm{MHz}$.

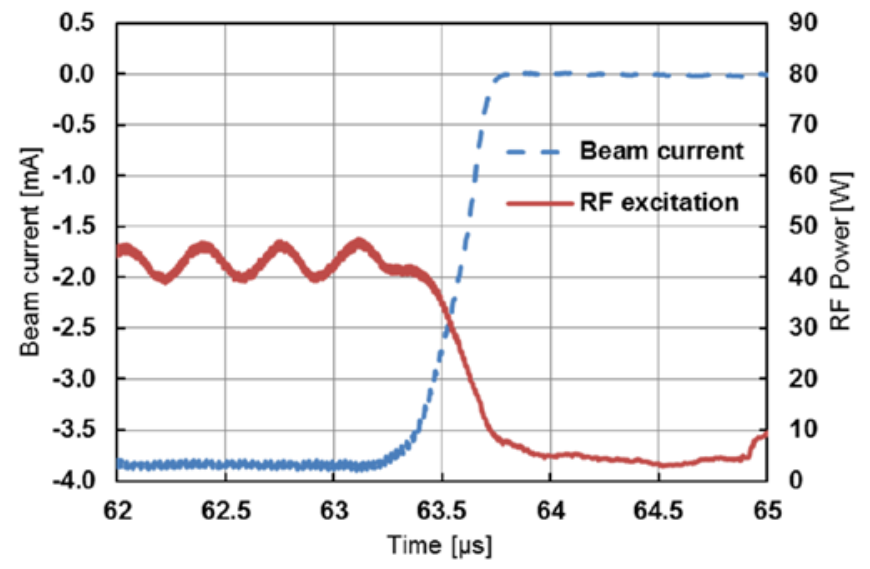

Fig. 8. The electron beam is switched off in $800 \mathrm{~ns}$ by modulation of the RF signal.

Figs. 6, 7 and 8 present the results obtained from the EB gun in a test at $-25 \mathrm{kV}$ accelerating voltage, $5 \times 10^{-5}$ mbar gun 
housing pressure, and 0.5 mbar plasma chamber pressure. First, a beam current of more than $3 \mathrm{~mA}$ was extracted from the plasma. Second, the results illustrate that the beam current can be controlled by modulation of the RF signal. Fig. 7 shows that the beam current is switched on in 200 ns using RF amplitude modulation. Fig. 8 shows that the beam current is switched off in $800 \mathrm{~ns}$. The minimum time to switch off is limited by the time for the plasma to extinguish and by the frequency of the RF signal [10].

\section{B. Comparison of the RF excited plasma-cathode with conventional electron sources.}

By using RF excitation to generate the plasma electron source, the plasma parameters can be rapidly changed compared to conventional control of diode EB guns, and materials processing triode guns. This leads to quicker and more accurate control of the beam parameters, not only over DC plasma-source guns, but also over thermionic-cathode guns. In some applications the beam is required to be switched on and off quickly and this would be feasible in less than 1 microsecond in an easy and inexpensive way. There is no waiting time for a metal filament to cool down, as in thermionic emitters; and no capacitance to be discharged as in DC excited plasma-source guns. As a result, the welding cycle is shortened [9]. An RF off-the-shell power supply can be used since 50 to $100 \mathrm{~W}$ are needed. The use of an RF signal would offer additional benefits such as beam power control by amplitude modulation and average beam power control by pulse-width modulation.

However, minimum beam pulse duration is determined by the plasma lifetime. At atmospheric pressure this is around 20 ns since the ionized species are recombined with ambient gas molecules. At low pressure this time can be several milliseconds. The plasma chamber diameter has been kept small, so that the ions collide against the walls and recombine more quickly, leading to the plasma extinction when the excitation has been stopped. Preferably, the plasma chamber dimensions are selected to be less than the average path length of the ions in the plasma, in order to ensure that the plasma lifetime is short after the RF excitation is stopped.

As a result, there is no grid electrode needed to control the beam current, and the gun can be implemented as a diode, producing high integrity particle beams. In addition, the power supply and control units are simpler and less expensive than for conventional EB guns. This may contribute to a reduction in size of the equipment. Since cathode is not damaged due to particle bombardment as with metallic filaments, it might be possible to operate the device at higher pressures, and thus to have a simpler pumping system for evacuating the EB gun chamber.

In thermionic cathodes, filament thickness and surface properties change during the cathode lifetime. In addition, when the filament is replaced differences in the beam quality and the device performance can become apparent. Using a plasma cathode as the electron source avoids these problems and provides an unlimited cathode life that has no wear giving a repeatable and uniform performance, which is very important in material processing [1].

\section{CONCLUSION}

A plasma cathode EB gun with a number of novel features has been presented. Since there is no filament to wear out, maintenance and beam distortion problems have been substantially reduced compared with thermionic electron guns. Modulation of the RF power can be used to control the beam intensity, such that a grid electrode is not required, avoiding beam aberration. Moreover, the plasma cathode EB gun can readily produce rapidly modulated beam powers utilizing much less complex power supply systems.

Applications of the RF plasma EB gun are cutting, welding, curing, melting, additive layer manufacturing, drilling, and gas treatment, among others.

A need to understand the plasma that is generated in the apparatus was identified and work on the plasma diagnosis is ongoing. A separate setup for the study of the RF generated plasma was developed.

\section{REFERENCES}

[1] H. Schultz, Electron Beam Welding. Abington Publishing, Cambridge, UK, 1993.

[2] O. Richardson, Science, 38(967), pp. 57-61.

[3] G Fiksel et al., Plasma Sources Sci. Technol., 5, pp.78-83, 1996.

[4] J. Pierce, Theory and design of electron beams, 2nd ed. New York, 1954.

[5] N. Rempe et al., Welding and Cutting, 11(2), 2012.

[6] S.I. Belyuk et al., Russian Physics Journal, 44(9), pp. 987-995, 2001.

[7] A.S. Bugaev et al., Hardware Laser and Particle Beams, 21, p. 139, 2003.

[8] S. Yu. Kornilov et al., Instrum. Exper. Tech., 52(3), pp. 406, 2009.

[9] S.I. Belyuk et al., "Electron-beam welding guns with plasma cathodes," Avt. Svarka, no. 1, pp. 49-50, 1974.

[10] C.N. Ribton et al., "Plasma source apparatus and method for generating charged particle beams”, UK Patent Application, Patent GB1210607.6, 2012.

[11] E.M. Oks, Plasma Sources Sci. Technol. 1, 249, 1992.

[12] S. Humphries, Charge Particle Beams, p.321, 1990.

[13] Tuohimaa T, Liquid-Jet-Target Microfocus X-ray Sources: Electron Guns, Optics and Phase-Contrast Imaging. Royal Institute of Technology. Stockholm, Sweden, 2008. 


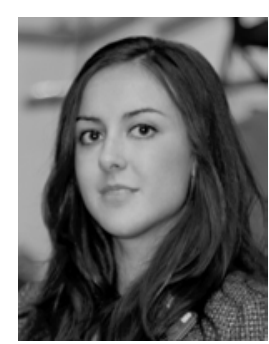

Sofia del Pozo (GSMIEEE'12) was born in Toledo, Spain, in 1990. She received the B. Eng. degree with honors in industrial engineering in electronics from Castilla-La Mancha University, Toledo, Spain, in 2011, and the M. Sc. degree in advanced engineering design from Brunel University, London, UK, in 2012.

She is currently pursuing the Ph.D. degree in electronic and computer engineering with Brunel University and she is based for her research at The Welding Institute (TWI) Ltd, Cambridge, UK. She has been working on the design, development and testing of a thermionic-cathode electron beam gun for the treatment of marine engine emissions as part of the DEECON project (FP7 programme). Her research interests include electron beam gun design, plasmas as electron sources, FE analysis, plasma diagnosis, and spectroscopy.

Ms. del Pozo is a student member of the Institute of Physics (AMInstP) and the Welding Institute (AWeldI), and she was awarded the 'Alumni Prize for Advanced Engineering Design' for her Masters project by Brunel University in 2013.

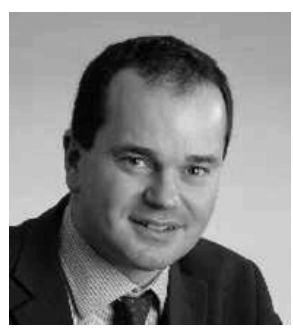

Colin N. Ribton graduated from The University of Nottingham in 1984 with a joint honors degree in pure and applied physics. He joined TWI's Electron Beam group in 1985. In 2001 he left TWI to join a company developing novel antenna technologies, where he became Vice President of Application Engineering. He re-joined

TWI in 2003.

His roles at TWI have involved him in the computer modeling of electron optics and high voltage components, the design of high voltage power supplies, the design and optimization of radiation shielding, real-time control system architecture, and the design of digital and analogue electronics. In particular, this has been involved in the development of equipment and processes to manufacture major components in power generation, nuclear, aerospace and medical applications. He is presently a Technology Consultant in the EB group where he is active in promoting EB technology for new applications.

Mr. Ribton is a Chartered Physicist (CPhys), a Chartered Engineer (CEng), a Member of the Institute of Physics (MInstP), and a Member of the Welding Institute (MWeldI).

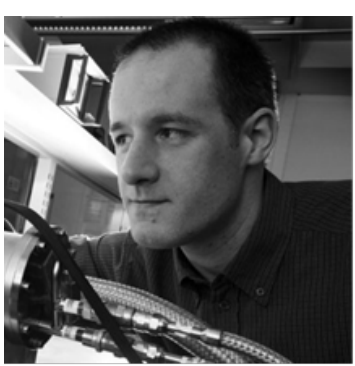

David R. Smith, received the M.Phys. honours degree in physics with space science and systems from the University of Kent, Canterbury, Kent, U.K., in 2000, and the Ph.D. degree from the University of Leicester, Leicester, U. K., in 2004. His Ph.D. thesis was entitled "Radiation damage in charge-

coupled devices.”

He has continued to do research in the field of radiation dosimetry and radiation damage in silicon devices and is currently a Senior Lecturer in the School of Engineering and Design at Brunel University, Uxbridge, U.K. He has published a number of papers on the effects of proton damage on CCD operation, in particular the generation and characteristics of bright pixels exhibiting "random telegraph signal" behaviour, and the effects of radiation damage on the operational characteristics of electron-multiplication CCDs.

Dr. Smith is a Member of the Institute of Physics, a Fellow of the British Interplanetary Society and a Fellow of the Higher Education Academy. 